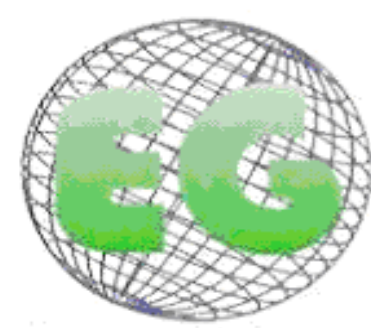

ISSN 1695-6141 N'25.

\title{
Consideraciones generales en la atención de urgencia a la paciente obstétrica politraumatizada
}

General considerations emergency care in the obstetric patient polytrauma

*Mejías Paneque, C., **Duarte González, L., ***García González, S.
* Matrona Hospital Virgen Macarena. **Matrona Atención Primaria. ***Matrona Hospital Juan Ramón. Jiménez.Huelva.

Palabras clave: : embarazo; politraumatismo; reanimación cardiopulmonar

Keywords: pregnancy, multiple trauma, cardiopulmonary resuscitation.

\section{RESUMEN}

El presente artículo tiene como objetivo principal que los sanitarios conozcan las modificaciones fisiológicas que se producen en la mujer embarazada y que precisan consideraciones especiales en la atención de urgencias prehospitalaria para conseguir mejores resultados materno-fetales.

Para su elaboración se ha procedido a una búsqueda sistemática de las principales bases de datos: Cinahl, Pubmed, Cuiden, Cochrane, Google académico y Revista Matronas Profesión con las palabras clave: Politraumatismo y Embarazo. La búsqueda obtuvo un resultado de 55 artículos de los cuales se eliminaron 33 por su falta de validez en la metodología o no estar relacionados con el objeto de estudio.

Como conclusión podemos decir que el aumento de la actividad laboral de la mujer, la persistencia en su trabajo hasta el final de su gestación y el mayor uso del automóvil han incrementado las tasas de traumatismos obstétricos y de la morbi-mortalidad de la mujer en los países industrializados ${ }^{(1)}$ siendo la consecuencia más importante la muerte fetal ${ }^{(2)}$, es necesario conocer las modificaciones fisiológicas propias del embarazo y su repercusión en la compensación orgánica para proporcionar la mejor atención posible y mejorar los resultados maternos así como garantizar el mayor bienestar fetal.

\section{ABSTRACT}

This article's main objective is that the toilets are aware of the physiological changes that occur in pregnant women who require special considerations in prehospital emergency care for better maternal and fetal outcomes. 
Its production has made a systematic search of major databases: Cinahl, Pubmed, Take care, Cochrane, Google Scholar and Midwifery Profession Magazine with keywords: trauma and pregnancy. The search yielded a score of 55 articles of which 33 were eliminated for lack of validity in the methodology or may not be related to the subject matter.

In conclusion we can say that the increase in female labor activity, persistence in his work until the end of gestation and greater car use have increased rates of obstetric trauma and morbidity and mortality of women in the industrialized countries (1) being the most important consequence of fetal death (2), it is necessary to understand the physiological changes of pregnancy and their own impact on the organic compensation to provide the best care possible and to improve maternal outcomes and ensure the greater good fetal.

\section{INTRODUCCIÓN}

Las modificaciones fisiológicas que se producen en la mujer como consecuencia de la gestación hacen que las medidas generales de urgencia aplicadas al resto de la población no sean útiles en estos casos. ${ }^{(3)}$

Saber realizar una adecuada valoración de la gestante traumatizada así como un correcto manejo de la situación nos ayudará a minimizar las repercusiones del accidente sobre la madre y el feto, mejorando su supervivencia. ${ }^{(4,5)}$

Hoy día sabemos que la reanimación de una embarazada traumatizada debe centrarse en la madre porque la causa más común de muerte fetal es el shock materno, el cual puede no manifestarse hasta que la madre ha perdido el $30 \%$ de su volumen sanguíneo por la hipervolemia del embarazo, por lo tanto, no es recomendable perder el tiempo en la auscultación fetal en la escena del trauma ya que puede resultar dificultoso y retrasar la atención a la madre. Así, en la escena del accidente consideraremos que el feto está vivo hasta que llegue al hospital o podamos proceder a auscultación fetal con doppler tras estabilización materna ${ }^{(6)}$.

Es lógico pensar que en el caso de embarazadas conscientes, la victima sumará a su propia angustia por el accidente el temor por el bienestar fetal, esto hará que el equipo de emergencias tenga que tranquilizar a la madre ofreciéndole apoyo y confianza y recordándole que el mejor tratamiento para el feto es el tratamiento de su propia madre.

\section{OBJETIVO}

Que los sanitarios conozcan las modificaciones fisiológicas que se producen en la mujer embarazada y que precisan consideraciones especiales en la atención de urgencias prehospitalaria para conseguir mejores resultados materno-fetales.

\section{METODOLOGÍA}

Se procede a revisar la evidencia científica actual sobre el manejo de la paciente obstétrica politraumatizada. Como criterios de inclusión para la selección de artículos a revisar se ha tenido en cuenta que la publicación se haya realizado en los últimos dieciséis años, (siendo el artículo más antiguo de 1994), debido a la escasez de publicaciones respecto a este tema. Por otro lado hemos descartado todos aquellos artículos que no hayan tenido una buena calidad metodológica en su elaboración. Los idiomas utilizados en los artículos eran el español y el inglés. 
Para su elaboración se ha procedido a una búsqueda sistemática de las principales bases de datos: Cinahl, Pubmed, Cuiden, Cochrane, Google académico y Revista Matronas Profesión con las palabras clave: Politraumatismo y Embarazo.

\section{RESULTADO}

La búsqueda obtuvo un resultado de 55 artículos de los cuales se eliminaron 33 por su falta de validez en la metodología o no estar relacionados con el objeto de estudio.

\section{IMPLICACIONES PARA LA PRÁCTICA CLÍNICA}

Tras la revisión bibliográfica podemos extraer las siguientes consideraciones generales de actuación específicas para la paciente obstétrica y que son diferentes a las de la población general por la fisiología propia del embarazo:

\section{1.- VALORACIÓN PRIMARIA.}

Como punto inicial de partida debemos considerar la posibilidad de que toda mujer en edad fértil puede estar embarazada. En el caso de que la gestación sea evidente, es fundamental datar la gestación pues esto determinará la viabilidad del feto y posibles complicaciones gravídicas que podemos encontrarnos, para ello podemos utilizar el tamaño del útero para datar la edad gestacional: A las 20 semanas de gestación el útero alcanza la altura del ombligo, creciendo alrededor de un centímetro por cada semana de embarazo ${ }^{(7)}$. Así si una gestante presenta una altura uterina de $5 \mathrm{~cm}$. por encima de su ombligo, podremos decir que la gestación está en torno a la vigesimoquinta semana de gestación.

Determinaremos el estado ventilatorio y cardiovascular. Si está indicada la intubación debemos tener presente el mayor riesgo de broncoaspiración debido al retraso en el vaciamiento gástrico de la embarazada.

Siempre se administrará oxígeno a alto flujo debido a que el consumo de oxigeno esta aumentado en la embarazada ${ }^{(8)}$, considerando incluso la posibilidad de hiperventilar a la mujer si es necesario. En el caso de necesitar colocar un tubo endopleural debemos considerar que en la gestante el diafragma asciende unos $4 \mathrm{~cm}$. por lo que su colocación se realizará en el 3 o 4 espacio intercostal.

La hipovolemia debe ser tratada con soluciones Cristaloides o Suero Fisiológico $0.9 \%$, siendo adecuada una reposición de líquido de 3:1 (cristaloides: sangre perdida) con lo que mejoraremos la perfusión uteroplacentaria. Está totalmente contraindicado el uso de aminas vasoactivas $^{(9)}$. Considerar que ante una hemorragia activa debido a la hipervolemia de la embarazada, ésta es capaz de conservar TA y pulso normales hasta el momento de entrar en fracaso hemodinámico franco.

Respecto a la posición de la paciente, la premisa "se actuará como si existiesen lesiones de columna vertebral y/o medular con un cuidado extremo a nivel cervical mientras no se demuestre lo contrario" es también aplicable a la embarazada, si bien en gestante de más de 20 semanas para evitar el "efecto Poseiro" (repercusiones hemodinámicas debido a la compresión uterina sobre la vena cava inferior) debemos colocarla de decúbito lateral izquierdo. Por lo tanto, si la paciente presenta un alto nivel de consciencia que nos haga descartar las lesiones anteriores la colocaremos en posición de seguridad con inclinación lateral hacia la izquierda; si no es posible descartar estas lesiones procederemos a ${ }^{(10)(11)}$ : 
a) Utilización de tablero espinal: Una vez inmovilizada con las correspondientes contenciones cefálicas y almohadillado, así como firme sujeción, colocaremos cuñas en el lado derecho para lograr un decúbito lateral izquierdo de $30-40$ sobre la horizontal.

b) Si no disponemos de tiempo para la colocación anterior (por ejemplo, precisamos realizar RCP) manualmente un miembro del equipo desplazará el útero hacia la izquierda.

Se evitará en lo posible canalizar la vena femoral, pues la obstrucción de la vena cava inferior por la presión del útero la convierten en una vía poco eficaz.

En el caso de necesitar utilizar un pantalón antishock, nunca utilizaremos la cámara abdominal pues disminuirá el flujo utero-placentario comprometiendo el estado fetal.

Finalmente hablaremos de los tiempos: El tiempo óptimo para una resucitación exitosa de un adulto no gestante es menos de 5 minutos de apnea o ausencia de pulso. Sin embargo, el feto de una madre apneica y sin pulso tiene 2 minutos o menos de reserva de oxígeno, debido a que la tensión de oxígeno en la vena umbilical siempre es menor que 4 en la vena uterina. Entonces, la duración tolerable de apnea y/o ausencia de pulso de una mujer gestante es sólo de 4 minutos. Las posibilidades de una resucitación exitosa de una madre o feto disminuyen después de este tiempo.

\section{2.- VALORACION SECUNDARIA}

Una vez realizada la valoración inicial e iniciadas las maniobras de $\mathrm{RCP}$, si fuesen necesarias, procederemos a una valoración secundaria centrándonos en:

a) Valorar irritabilidad y dolor uterino. Las contracciones uterinas tetánicas pueden indicar desprendimiento de placenta.

b) Contracciones uterinas subjetivas de inicio de trabajo de parto prematuro.

c) Examen vaginal, lo ideal es que sea realizado por un obstetra para valorar la presencia de líquido amniótico subjetivo de ruptura de bolsa de líquido amniótico o sangrado vaginal.

d) Debido a las modificaciones fisiológicas ocasionadas por el embarazo es especialmente importante la reevaluación periódica de la embarazada pues pueden sufrir descompensaciones muy rápidas del estado hemodinámico.

e) Toda gestante de más de 20 semanas de gestación que ha sufrido un traumatismo grave requiere monitorización continua durante un mínimo de 4 horas.

f) Toda gestante que sea $\mathrm{RH}$ - se le debe poner gammaglobulina anti - $\mathrm{D}{ }^{(12)}$ dentro de las primeras $72 \mathrm{~h}$. postraumatismo.

Finalmente debe considerarse la posibilidad de ingresar a la mujer en un Hospital maternal de 3 Nivel cuando la paciente presente sangrado vaginal, hipovolemia o cambios en los tonos cardíacos fetales.

\section{DISCUSIÓN Y CONCLUSIONES}

Existe una clara escasez de artículos que traten sobre casos clínicos en pacientes politraumatizadas, esto puede hacer que muchos sanitarios al encontrarse con una 
embarazada politraumatizada centren su atención en el embarazo y en el bienestar fetal, lo cual supone un claro error pues el bienestar fetal será directamente proporcional al bienestar materno.

Es necesario conocer las modificaciones fisiológicas propias del embarazo y su repercusión en la compensación orgánica para proporcionar la mejor atención posible y mejorar los resultados maternos así como garantizar el mayor bienestar fetal.

\section{BIBLIOGRAFÍA}

1.- Macías Seda J, Álvarez Gómez J.L, Orta M. A. Traumatismos en la embarazada. Puesta al día en Urgencias, emergencias y catástrofes 2000; 1(4): $237-245$.

2.- European Trauma care course. Trauma in pregnancy. Trauma ORG. 1998.

3.- Kuhlman RS, Crukshank DP. Maternal trauma during pregnancy. Clin Obstet Gynecol, 1994; 37: 74-293.

4.- Pimentel L. Mother and Child: Trauma in pregnancy. Emerg Med Clin North Am; 1991; 9: 549-561.

5.- Dominguez F. M. Traumatismo durante el embarazo. Principios de urgencias, emergencias y cuidados críticos. Uninet. Disponible en: http://www.uninet.edu/tratado/c1108i.html. Fecha de consulta 10/08/2011.

6.- Glossman NB. Blunt Trauma in Pregnancy. American Family Physician, 2004. Volumen 70, Number 7.

7.- Usandizaga JA, De la Fuente. Tratado de Obstetricia y Ginecología. Volumen 1: Obstetricia. Madrid: McGraw-Hill, Interamericana, 2005.

8.- Franco Utili. Programa de Medicina de Urgenica. P. Universidad Católica. Disponible en: http://www.urgenciauc.com/profesion/pdf/Trauma Embarazo.pdf. Fecha de consulta 20/08/2011.

9.- Neil J. Murphy, Sue Redd N. Resucitación maternal y trauma. Also, 2008.

10.- Lowdermilk. Perry Bobak. Traumatismo durante el embarazo. Enfermería maternoinfantil. Enfermería Mosby 2000. Barcelona: Mosby; 1981. Pg 920 - 930.

11.- Moratal, R. Módulo obstetric. Manual de Protocolos en extrahospitalaria. Emergencias, 2a Edición. Madrin: Aran, 2002.

12.- Pearlman MD, Tintinalli JE. Evaluation and treatment of the gravida and letus following trauma during pregnancy.

Obstet Gyneeol Clinics 01North Ame r 1991;18(2):371-381. (Level111) 


\section{ANEXO:}

TABLA 1: PROTOCOLO BÁSICO DE TRATAMIENTO

\begin{tabular}{|l|l|}
\hline 1 & Realiza medidas de soporte vital básico y avanzado. \\
\hline 2 & Controlar hemorragia. \\
\hline 3 & Realizar inmovilización de la zona que precise. \\
\hline 4 & $\begin{array}{l}\text { Administrar perfusión hemodinámica: líquidos ( fundamentalmente } \\
\text { cristaloides y Solución Fisiológica al 0.9\%), sangre. }\end{array}$ \\
\hline 5 & $\begin{array}{l}\text { Controlar constantes: TA, pulso, respiración. Reevaluación continua } \\
\text { del estado de la gestante por descompensaciones bruscas del estado } \\
\text { hemodinámico. }\end{array}$ \\
\hline 6 & $\begin{array}{l}\text { Colocar en posición de seguridad en decúbito lateral izquierdo para } \\
\text { prevenir el síndrome de la vena cava y favorecen perfusión utero- } \\
\text { placentaria. }\end{array}$ \\
\hline 7 & $\begin{array}{l}\text { Administrar oxígeno si la T.A es baja y la respiración forzada ( } \\
\text { asegurar siempre saturación de Oxígeno > 90 \% utilizando oxígeno a } \\
\text { altas concentraciones) }\end{array}$ \\
\hline 8 & Realizar los preparativos para la cesárea de urgencia si es necesario \\
\hline
\end{tabular}

